Monatsschrift f. Geburtshülfe u. Gynäkologie 1915;42:I-IV

\title{
Contents, Vol. 42, 1915
}

\section{Inhalts -Verzeichnis.}

Original-Arbeiten. seite

Amann, J. A., Ueber Fibroadenoma fornicale (Serositis

fibroa denomatosa recto-cervicalis) 492

Bauch, B., Zur Frage der Leberfunktionsstörung während der Gravidität 258

Baumann, E r w i n, Die Erfahrungen mit der Abder-

haldenschen Schwangerschaftsdiagnostik im Frauenspital

Basel 199

Benthin, W., Zur Kritik der Behandlung des febrilen

Abortes 162.

Boldt, H. J., Intermediäre Trachelorrhaphie als Prophy-

laktium gegen die mitunter auftretenden unangenehmen

Ergebnisse infolge von Cervixrissen 372

Frankl, Oskar, Plexus venosus varicosus endometrii 139 Hiissy, Paul, Wehenschwäche und

Wehenmittel . . . 477 Ingerslev,E·, Notizen über die Wertschätzung und Be-

nutzung der Geburtszange im ersten halben Jahrhundert

nach ihrer Einführung in die Geburtshülfe 511

Kehrer, E., Erfahrungen über Sakralanästhesie besonders

bei gynäkologischen Operationen. (Hierzu Tafel III) . . 95

, Symphysenlockerung und Symphysenruptur .... 321

$\mathrm{L}$ a u t h, Gu s t a v, Ueber das Verhalten des Uterus bei

ovariellen Blutungen 36

Mandl, Ludwig, Ueber seltenere Indikationen zum

Kaiserschnitt 499

Mayer,A., Ueber Parametritis und Paravaginitis posterior

mit heterotoper Epithelwucherung (Adenomyositis uteri

et recti) $\quad 403$

Prochownik, L., Gynäkologie und Pankreas 241

Runge, Ernst und Ernst Gruenhagen, Zur

röntgenologischen Beckenmessung 292

Schindler, Rudolf, Histologische Untersuchungen an

mit intravenösen Injektionen kolloidaler Metalle behan-

delten Fallen 389

Schweitzer, B., Entstehung, Verhíitung und Behandlung der artefiziellen Uterusperforationen bei Abort .... 148 Schultze,B. S., Zur Frage der inneren Untersuchung mit 
Seedorff, M., Ein Fall von geborstener Ovarialgravidität 30 Thaler, Hans, Zur Anwendung der Lokalanästhesie bei größeren vaginalen Operationen 438

-, - und Hans Zuckermann, Zur Prophylaxe endogener Wochenbettfieber mittels $5 \%$ Milchsäurespülungen während der Schwangerschaft. (Hierzu Tafel I-II) 1, 181

- IV -

Seite

Velde, T h. van de, Geburtsstörungen durch Entwick-

lungsfehler der Gebärmutter 307

Waasbergen, G. H. van, Zur Abderhaldenschen Fer-

mentreaktion bei Nichtschwangeren 230

Feldgraue Geburtshülfe 1914/15 81

Nekrologe $\quad 82,181,184,186,187,543$

Selbstbesprechungen.

Längenmessung der weiblichen Urethra. Von L. S a 1 m o n y 279 Strahlenbehandlung in der Gynäkolosíe. Von T h. H. van de

Velde ' 281

Beriehte.

Referat über die italienische gebvrtshülflich-gynäkologische

Literatur 1914. Von Dr. PaulHüssyin Basel ... 46

Die geburtshülflich-gynäkologischen Dissertationen der deutschen Universitäten (Winter-Semester 1914/15). Ref. von

Priv.-Doz. Dr. F. Weber in München 67

Tagesfragen.

Die deutsche Gesellschaft für Bevölkerungspolitik. Von Dr.

M. Hofmeier in Würzburg 539

Internationale Gynäkologen. Von A. Martin 464

Perforation vnd Strafgesetzreform. Von Dr.HansLieske

in Leipzig 270

Tagesnachrichten und Personalien $\quad 187,282,471$

Vereins- und Literaturbeilagen.

Mtinchener gynäkologische Gesellschaft. Sitzung am 20. V.

$1915 \quad 547$

Referaíe aus geburtshülflich-gynäkologischen Geseilsehaften

85, 188, 283, 376, 472, 551 Geburtshülflich-gynäkologisehe Mitteilungen aus Medizinisehen

Geseilsehaften und Vereinen . 85, 188, 283, 376, 472, 552

Buchbesprechungen 86, 188, 284, 377, 553

Druckfehlerberiehtigung

Literaturverzeichnis $92,195,285,386,473,560$

Sachregister 563

Namenregister 566 